\title{
Laser Annealing of Amorphous NiTi Shape Memory Alloy Thin Films to Locally Induce Shape Memory Properties
}

\author{
X. Wang ${ }^{\text {a }}$, Y. Bellouard ${ }^{\text {b,1 }}$, J. J. Vlassak ${ }^{\mathrm{a}, *}$ \\ ${ }^{a}$ Division of Engineering and Applied Sciences, Harvard University, Cambridge, MA 02138, U.S.A \\ ${ }^{b}$ Center for Automation Technologies / Center for Integrated Electronics, Rensselaer Polytechnic Institute, Troy, \\ NY 12180, USA
}

\begin{abstract}
We present the results of a crystallization study on NiTi shape memory thin films in which amorphous films are annealed by a scanning laser. This technique has the advantage that shape memory properties can be spatially distributed as required by the application. A kinetics study shows that nucleation of the crystalline phase occurs homogenously in the films. Consequently, the laser annealing process produces polycrystalline films with a random crystallographic texture. The crystallized films have a uniform microstructure across the annealed areas. The material in the crystalline regions transforms reversibly to martensite on cooling from elevated temperature and stress measurements show that a significant recovery stress is achieved in the films upon transformation.
\end{abstract}

Keywords: Shape memory alloy thin films; Laser annealing; Crystallization kinetics; Martensitic phase transformation

\footnotetext{
${ }^{1}$ Present address: Micro- \& Nano-Scale Engineering, Mechanical Engineering, Eindhoven University of Technology, PO Box 513, 5600 MB Eindhoven, The Netherlands.

${ }^{*}$ Corresponding author. Tel.: +1 617496 0424; fax: +1 617495 9837. E-mail address: vlassak@esag.harvard.edu (J.J. Vlassak)
} 


\section{Introduction}

Shape Memory Alloys (SMAs) are active materials that derive their unique properties from a thermoelastic martensitic transformation. They have been studied extensively over the last fifty years with most attention focused on bulk materials. Recently, the shape memory effect has been demonstrated in thin films of these alloys [1-7], making them attractive candidates for use as actuators in microelectromechanical systems (MEMS). It is, however, difficult to induce an intrinsic two-way shape memory effect in thin films and a biasing spring is generally needed to restore the initial state after actuation. As a result, use of SMA actuators in MEMS has been limited to bimorph-like mechanisms. The technique of Laser Annealing of Shape Memory Alloys (LASMA) recently emerged as a promising approach for the fabrication of planar mechanisms [8]. Using this technique, shape memory properties can be spatially distributed across a material: crystallized material has shape memory properties and can be used as an actuator, untransformed material is passive and provides the restoring force. Various aspects of the LASMA process, especially for thin films, have yet to be explored. In this paper, we present the results of a crystallization study in which a laser was used to crystallize selected areas of amorphous NiTi films.

\section{Experimental}

NiTi thin films with a thickness of approximately $1.5 \mu \mathrm{m}$ were deposited on $1 \mathrm{~mm}$ thick fused quartz substrates by dc magnetron sputtering. The background pressure of the sputter 
chamber was less than $5 \times 10^{-8}$ Torr; the pressure of the Ar working gas was 1.5 mTorr. All depositions were performed at room temperature. The films were grown by co-sputtering an equiatomic Ti-Ni alloy and an elemental Ti target. The composition of the films was controlled by varying the power to individual guns [7]. The nominal target-substrate distance was $100 \mathrm{~mm}$ and the deposition rate was approximately $0.3 \mathrm{~nm} / \mathrm{s}$. During deposition, the substrates were rotated at a speed of 20 RPM to maintain film thickness and composition uniformity. The composition of the films was measured to be 50.5 \pm 0.2 at\%Ti using Electron Microprobe Analysis (EMPA). X-ray diffraction (XRD) and high-resolution transmission electron microscopy (HRTEM) confirmed that the structure of the as-deposited films was entirely amorphous.

In order to crystallize the NiTi films, samples were annealed by scanning a laser beam over the surface of the films. A fiber-injected CW high power near-IR laser diode (coherent/925 $\mathrm{nm})$ was used in this study. The laser beam had a Gaussian power distribution and a diameter of approximately $0.9 \mathrm{~mm}$ (i.e., the diameter at 1/e intensity) as determined using the knife-edge approach. The specimen was mounted on a platform with three degrees of freedom, capable of planar translational and rotational motions with micron resolution. The platform (Yaskawa, Robotworld) is fully programmable and its acceleration, speed, and position can be accurately controlled. A program was developed to define repeatable and reconfigurable annealing patterns. In the experiments, the laser power was varied from 5 to $9.4 \mathrm{~W}$; the scan speed was varied from 1 to $8 \mathrm{~mm} / \mathrm{sec}$. All scans were performed in air in a thermally stabilized environment. During laser annealing a thin oxide coating was formed on the NiTi films. The oxide thickness was determined from the reflectivity spectrum measured using a spectrophotometer (Jasco V-570 NUV/VIS/NIR) 
and was typically in the range of $50-100 \mathrm{~nm}$ for the laser annealing parameters used in this study [9]. As expected the oxide thickness increased with increasing laser power and decreasing scan speed. Oxide formation could of course be reduced by performing the experiments in an inert atmosphere. The microstructure of the films after laser annealing was investigated using Philips EM420 and JEOL 2010 transmission electron microscopes (TEM). TEM specimens were prepared by cutting $3 \mathrm{~mm}$ diameter discs from the samples. The discs were dimpled and the area of interest was thinned to electron transparency by ion beam milling with a $4 \mathrm{kV}$ argon beam. Multiple line scans were performed to create large crystalline areas. Samples for texture analysis were cut from these areas. X-ray diffractometry was used to investigate the texture of the films after laser annealing. During the texture measurements, the samples were heated to ensure that only the austenite phase was present. Pole figures were obtained for the $\{200\},\{110\}$ and $\{211\}$ reflections. In order to investigate the evolution of the residual stress in the crystallized region as a function of temperature, multiple line scans were performed to create arrays of parallel lines where the film was crystalline. A short dwell time was introduced at the end of each line scan to allow the sample to cool down between adjacent scans. Films with various volume fractions of crystalline material were produced by varying the laser annealing parameters and the line spacing. The annealing parameters and corresponding volume fractions of crystalline material are listed in Table 1. For each volume fraction, two sets of rectangular specimens (6 mm x $25 \mathrm{~mm}$ ) were cut from these large arrays, i.e., one set with the long edge of the specimen parallel to the laser scan direction (labeled as RD in the figures) and one set perpendicular to the scan direction (labeled as TD). The stress in the long direction of the specimens was measured using the substrate curvature 
technique [10]. Using this approach, the residual stresses both parallel and perpendicular to scan direction could be measured. Stresses were calculated using the well-known Stoney equation [11], assuming a Young's modulus of $72 \mathrm{GPa}$ and a Poisson's ratio of 0.16 for the fused quartz substrate.

\section{Results and discussion}

Fig. 1 compiles the optical and TEM observations of the structure of the annealed NiTi films as a function of laser power density and scan speed. At a given scan speed, the film transitions from amorphous to partially crystalline and eventually fully crystalline with increasing laser power. The TEM micrographs in Fig. 1 show the microstructure close to the center of the laser trace where the annealing temperature is the highest. At low laser power, only a few isolated grains are formed in an amorphous matrix. Once the laser power is large enough to fully crystallize the film, the microstructure at the center is approximately independent of laser power. A similar transition occurs when the scan speed is varied at constant laser power. If the laser power density is too large, film and substrate crack due to thermal shock. As a result of the Gaussian intensity profile of the laser, a temperature gradient is introduced in the film in the direction perpendicular to the laser trace. After the laser scan, a crystalline band a few hundred micron wide forms along the laser track. The width of the band increases with increasing power density and decreasing scan speed. Fig. 2 shows the microstructure in the direction normal to the scan. Again, grain size and distribution are the same across the entire crystalline region. Most grains are 1 to $1.5 \mu \mathrm{m}$ in diameter although there are a few grains as small as $0.3 \mu \mathrm{m}$. Combined 
with Fig. 1, Fig. 2 confirms that a uniform microstructure is formed in the crystallized film for the annealing parameters used in this study. Thus, the shape memory properties are expected to be uniform across the annealed areas also. This is certainly desirable when using these materials in MEMS applications.

Fig. 3 shows a typical room temperature XRD spectrum of a sample that underwent multiple-line scans and that is fully crystallized. After laser annealing, the material in the crystalline regions has transformed to martensite demonstrating that shape memory properties can be introduced using laser annealing. Some R-phase and untransformed parent phase are also present in the film at room temperature. No precipitates are observed in the laser-annealed films because of the short annealing times. The presence of the untransformed $\mathrm{B}_{2}$ phase may be related to the Ni-rich layer that forms immediately below the surface oxide [12]. Fig. 4 is a TEM micrograph of the martensite morphology most frequently observed in the films. Electron diffraction patterns show that $<011>$ type II twins are prevalent in the microstructure. This type of twin is also the most frequently observed twin in bulk materials [13] and furnace annealed NiTi thin films [14].

Fig. 5 shows a cross-section TEM micrograph of a partially crystallized film. Nucleation of the crystalline phase seems to occur homogenously in the film. The film surface and the film-substrate interface are clearly not preferential sites for nucleation. This is likely due to a small composition shift that occurs at these interfaces due to oxidation or reaction with the substrate [12]. These reactions result locally in slightly Ni-rich regions that have higher crystallization temperatures and activation energies $[15,16]$. The almost perfect circular shape of 
the crystals both in plan-view (Fig. 1) and in cross-section TEM (Fig. 5) indicates that their general three-dimensional shape is spherical. Evidently, grains grow isotropically until they impinge on each other or until they touch the film surface or the substrate. Fig. 6 (a) is a low magnification electron micrograph of an individual grain showing two sets of mutually perpendicular needle domains. The inset is a selected area diffraction pattern taken from both the matrix and needle domains. Since the rhombohedral distortion is very small, the pattern is indexed in terms of the B2 system for convenience. The inset shows a typical $[001]_{\mathrm{B} 2}$ type diffraction pattern of the R-phase with two sets of 1/3(110) superlattice reflections. Because a diffraction pattern taken from only the matrix shows $1 / 3(110)$ superlattice reflections in only the [110]* reciprocal lattice direction, the inset pattern indicates that the needle domains are twin-related to the matrix with $\{100\}_{\mathrm{B} 2}$ type twinning planes. The twinning planes also correspond to the traces of the domains. The growth interface between the crystal and amorphous matrix was investigated on an atomic scale by HRTEM image (Fig. 6(b)). Although the growth interface between the crystal and amorphous matrix looks smooth at low magnification, high-resolution TEM indicates that it actually consists of $\{100\}_{\mathrm{B} 2}$ and $\{110\}_{\mathrm{B} 2}$ planes. Similar growth morphologies have been found in partially crystallized $\mathrm{Ti}_{50} \mathrm{Ni}_{25} \mathrm{Cu}_{25}$ melt-spun ribbon [17]. Quantitative EDX measurements were performed across the growth interface (Fig. 7). Each data point is an average of more than 10 measurements at the same site and the results are listed in Table 2. The amorphous and crystalline phases share the same composition, indicating that the crystallization reaction is partitionless.

It is important to evaluate the crystallographic texture of laser annealed NiTi films because 
the recoverable strain depends on the texture of the films [18] and because strong textures may lead to anisotropic shape memory behavior [19,20], making the design and fabrication of actuators more challenging. A typical $\{110\}$ pole figure measured by X-ray diffraction is shown in Fig. 8. The laser scan direction is labeled as the rolling direction (RD) in the figure. The pole figure shows that the film is polycrystalline with a mostly random crystallographic texture. This is confirmed by the $\{200\}$ and $\{211\}$ pole figures (not shown). As a result of the uniform orientation distribution of the grains, the shape memory behavior is expected to be isotropic with similar behavior in both RD and TD directions. The random texture is consistent with the homogenous nucleation mechanism described above. If nucleation occurs homogenously inside an amorphous matrix, one would indeed expect no preferential orientation of the nuclei. For an elastically anisotropic material such as the NiTi, the residual stress in the amorphous coatings could possibly induce a texture in which grain orientations that are most compliant in the plane of the film dominate. Based on the single-crystal elastic constants of NiTi [21], this mechanism would result in a (100) fiber texture for the $B_{2}$ phase. Such a texture component is not observed in these films, however, indicating that the strain energy associated with the residual stress is not significant in defining the texture. This is consistent with a number of studies on the stress evolution during crystallization of amorphous NiTi films [7, 22], indicating a relatively low residual stress in the range of -200 to $200 \mathrm{MPa}$. The isotropic texture observed in this study is in good agreement with a study by Miyazaki et al [20] in which it was demonstrated that ex-situ annealing of amorphous NiTi films leads to a uniform orientation distribution of the grains. We argue here that this random texture is a consequence of the homogeneous nucleation mechanism by which the crystalline 
phase forms. The random texture should be contrasted with the strong $<110>$ fiber texture commonly observed in NiTi films sputtered at elevated temperatures where the films are crystalline as deposited [20]. Under these deposition conditions, the crystalline phase nucleates on the surface of the substrate naturally assuming an orientation that minimizes surface and interface energies.

As illustrated in Fig. 9, the average stress in the multiple-line specimens, $\sigma_{\text {average }}(T)$, depends on the stress in the crystalline and amorphous areas according to

$$
\sigma_{\text {average }}(T)=f \sigma_{\text {crystalline }}(T)+(1-f) \sigma_{\text {amorphous }}(T) \text {. }
$$

where $f$ is the volume fraction of crystalline material. The stress in the amorphous regions, $\sigma_{\text {amorphous }}(T)$, can be measured directly using the as-deposited samples; the stress in the crystalline regions is denoted by $\sigma_{\text {crystalline }}(T)$ and can be calculated using equation (1). The stress-temperature curves in the RD and TD directions are shown in Fig. 10. The stresses in both directions are the same over the range of annealing parameters used in this study indicating that an equi-biaxial stress state exists in the films. This is consistent with the random crystallographic texture and the uniform microstructure. The stress-temperature curves show a closed hysteresis loop as a result of the reversible martensitic transformation that occurs in these films. Upon cooling from elevated temperature, the film initially consists of austenite and any variation in stress is due to the thermal mismatch between the austenite and the substrate. On continued cooling, the martensitic transformation takes place and the stress relaxes to approximately 150 MPa at room temperature due to the self-accommodating nature of the martensite twin structure. On subsequent heating, the reverse transformation to austenite leads to a complete recovery of the 
residual stress in the austenite. The recovery stress ( 350MPa) is independent of the annealing parameters and represents the actuating capability of the film. The temperature hysteresis is quite small at approximately $20^{\circ} \mathrm{C}$. This is beneficial for a fast actuation response in practical applications. Slight variation of the transformation temperatures from sample to sample can be attributed to small differences in composition between the films. It is evident from the results in Fig. 10, that laser annealing indeed offers a technique for selectively crystallizing NiTi coatings and that the recovery stress can be used to actuate structures in a MEMS device.

\section{Conclusions}

In conclusion, we have investigated a laser annealing technique that allows us to selectively crystallize an amorphous Ti-Ni film in specific areas where shape memory properties are desired. The film undergoes homogenous nucleation and has a random crystallographic texture after crystallization. The crystalline films have a uniform microstructure across the annealed area for the range of laser annealing parameters used in this study. The material in the crystalline regions transforms to martensite at room temperature demonstrating that shape memory properties can be selectively introduced. Stress measurements show that a significant recovery stress is achieved in the laser annealed films making them useful materials for actuators in MEMS devices.

\section{Acknowledgements}

The authors would like to acknowledge financial support from the National Science 
Foundation (Grant DMR-0133559).

\section{References}

[1] Busch JD, Johnson AD, Lee CH, Stevenson DA. J Appl Phys 1990;68:6224.

[2] Walker JA, Gabriel KJ, Mehregany M. Sensors and Actuators A 1990;21-23:243.

[3] Ishida A, Takei A, Miyazaki S. Thin Solid Films 1993;228:210.

[4] Wolf RH, Heuer AH. J Microelectromech Syst 1995;4:34.

[5] Miyazaki S, Ishida A. Mater Sci Eng A 1999;273-275:106.

[6] Grummon DS, Zhang JP. Physica Status Solidi A 2001;186:17.

[7] Shih CL, Lai BK, Kahn H, Philips SM, Heuer AH. J Microelectromech Syst 2001;10:69.

[8] Bellouard Y, Lehnert T, Bidaux JE, Sidler T, Clavel R, Gotthardt R. Mater Sci Eng A 1999;273-275:795.

[9] Khelfaoui F, Bellouard Y, Gessmann T, Wang X, Vlassak JJ, Hafez M. In: Proceedings of the International Conference on Shape Memory and Superelastic Technology (SMST-2004). Germany 2004.

[10] Witvrouw A, Spaepen F. J Appl Phys 1993;73:7344.

[11] Stoney GG. Proc R Soc London A 1909;82:172.

[12] Wang X, Lai A, Vlassak JJ, Bellouard Y. Mater Res Symp Proc 2004;795:275.

[13] Knowles KM, Smith DA. Acta Metall 1981;29:101.

[14] Zhang JX, Sato M, Ishida A. Acta Mater 2001;49:3001.

[15] Buschow KHJ, J Phys F: Met Phys 1983;13:563. 
[16] Chen JZ, Wu SK. J Non-Cryst Solids 2001;288:159.

[17] Santamarta R, Schryvers D. Mater Trans 2003;44:1760.

[18] Shu YC, Bhattacharya K. Acta Mater 1998;46:5457.

[19] Inoue H, Miwa N, Inakazu N. Acta Mater 1996;44:4825.

[20] Miyazaki S, No VH, Kitamura K, Khantachawana A, Hosoda H. Int J Plasticity 2000;16:1135.

[21] Mercier O, Melton KN, Gremaud G, Hägi J. J Appl Phys 1980;51:1833.

[22] Fu YQ, Du HJ, Zhang S. Surface and Coatings Technology 2003;167:120. 
Tables

Table1 Multiple line laser annealing parameters for stress measurements

\begin{tabular}{|c|c|c|c|c|}
\hline Sample & $\begin{array}{c}\text { Laser power } \\
(\mathrm{W})\end{array}$ & $\begin{array}{c}\text { Scan speed } \\
(\mathrm{mm} / \mathrm{s})\end{array}$ & $\begin{array}{c}\text { Line spacing } \\
(\mathrm{mm})\end{array}$ & $\begin{array}{c}\text { Volume fraction of } \\
\text { crystalline material, } f\end{array}$ \\
\hline 1 & 6.91 & 3 & 0.6 & $50 \%$ \\
\hline 2 & 8.8 & 5 & 0.6 & $80 \%$ \\
\hline 3 & 7.55 & 4 & 0.4 & $90 \%$ \\
\hline 4 & 6.91 & 3 & 0.4 & $100 \%$ \\
\hline
\end{tabular}

Table 2 EDX analysis results

\begin{tabular}{|c|c|}
\hline EDX point & Ti (at.\%) \\
\hline 1 & $50.4 \pm 0.1$ \\
\hline 2 & $50.5 \pm 0.1$ \\
\hline 3 & $50.6 \pm 0.1$ \\
\hline 4 & $50.6 \pm 0.1$ \\
\hline 5 & $50.5 \pm 0.1$ \\
\hline
\end{tabular}




\section{Figures}

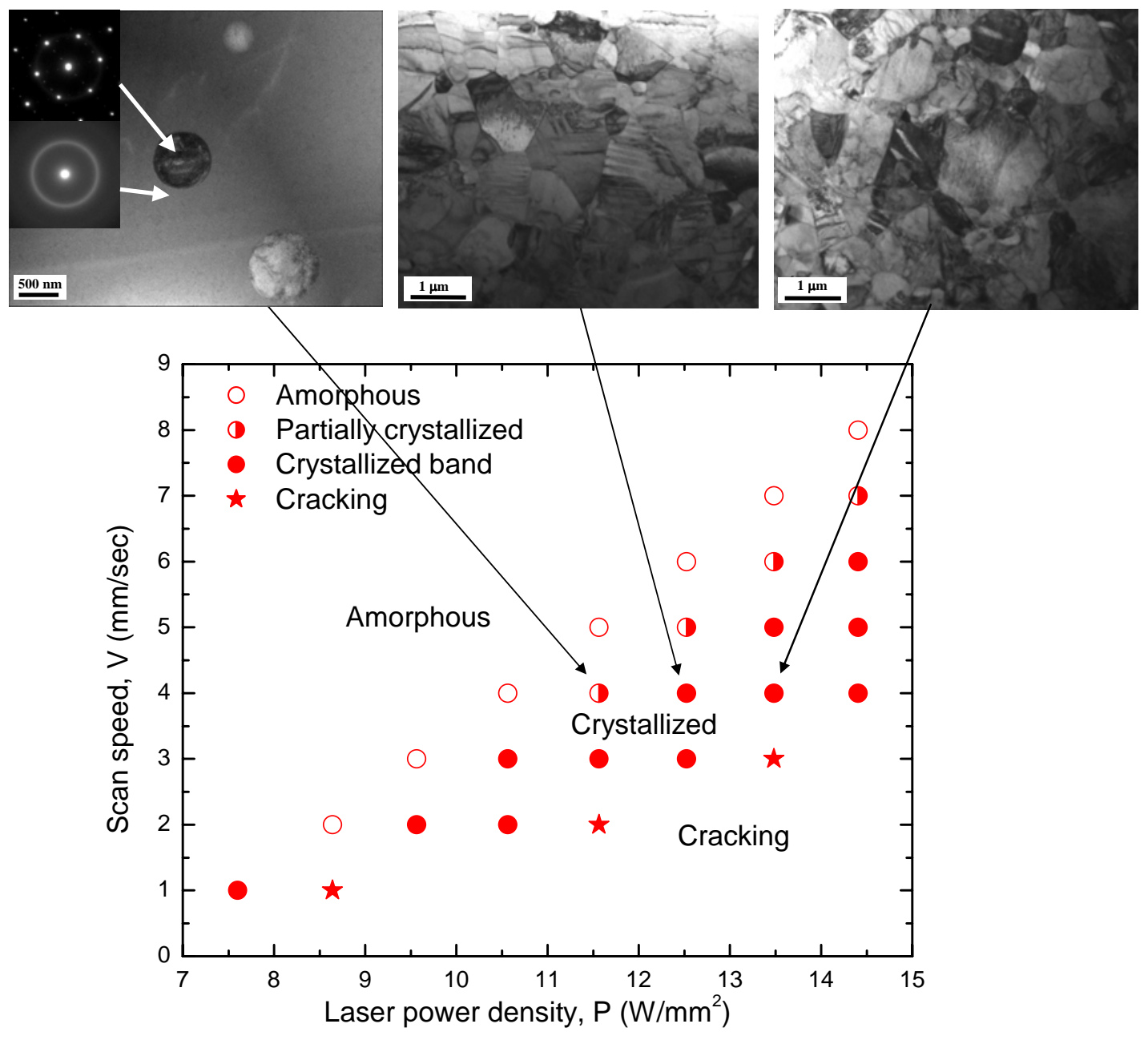

Fig. 1. Crystallization behavior of Ti-Ni films as a function of laser power density and scan speed based on optical (not shown) and TEM observations. 


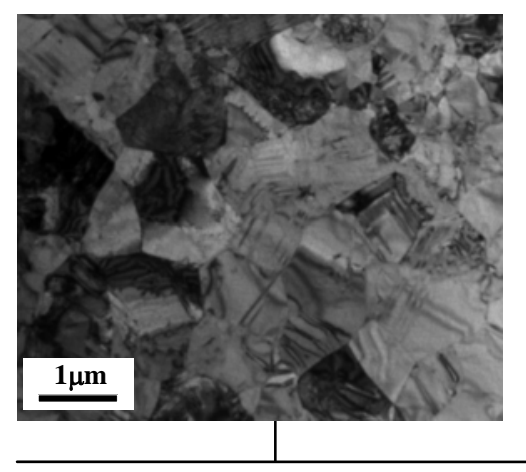

center

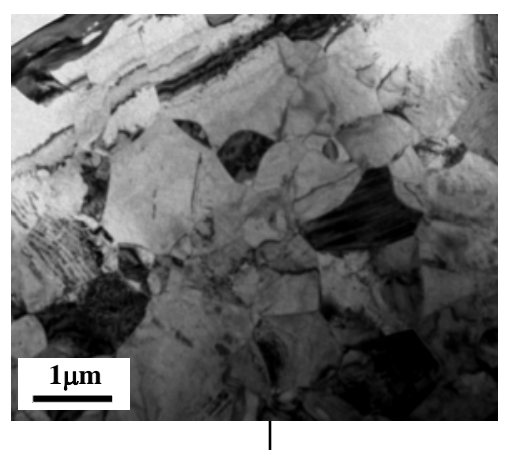

$\sim 100 \mu \mathrm{m}$

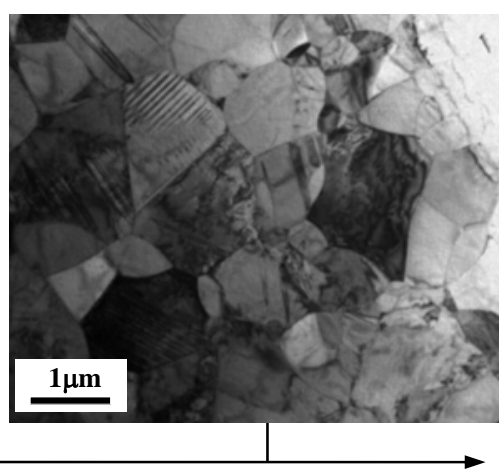

$\sim 200 \mu \mathrm{m}$

Fig. 2. The microstructure along the width of the crystallized band in a sample annealed with a laser power density of $14.4 \mathrm{~W} / \mathrm{mm}^{2}$ and a scan speed of $6 \mathrm{~mm} / \mathrm{s}$.

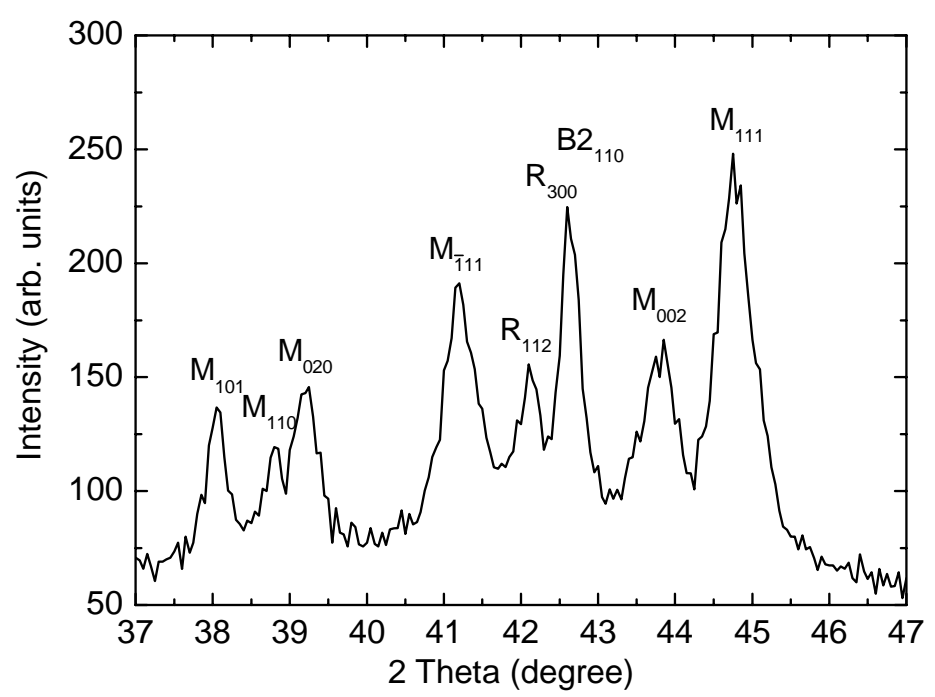

Fig. 3. X-ray diffraction spectrum for a sample with multiple laser scans. 

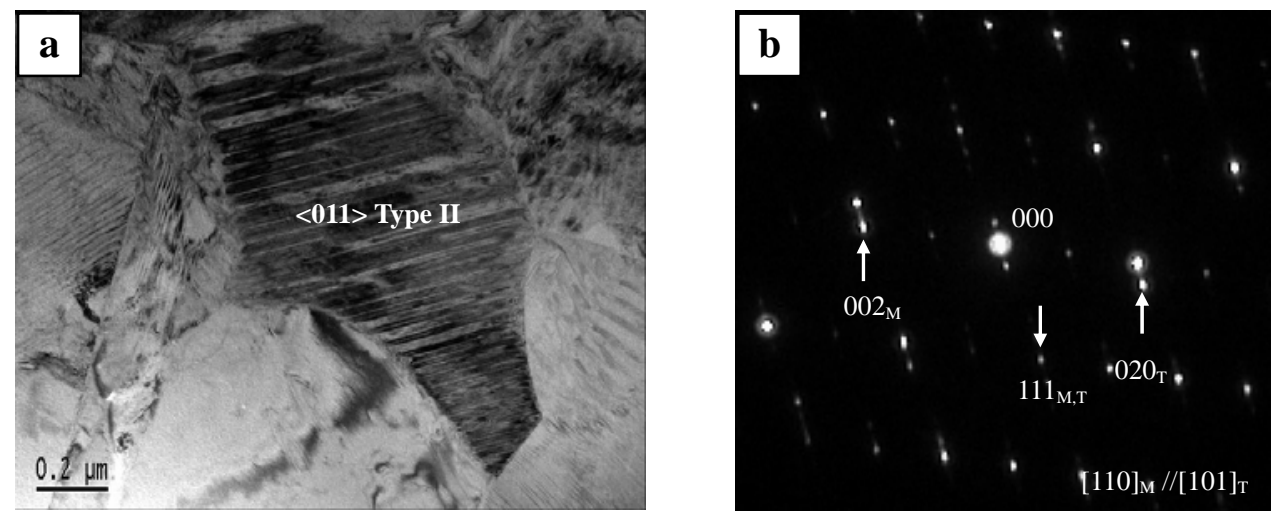

Fig. 4. (a) Bright field image of $<011>$ type II twins as main microstructure of martensite in the laser annealed NiTi films. (b) Electron diffraction pattern taken from the region, incident electron beam // [110 $]_{\mathrm{M}} / /[101]_{\mathrm{T}}$.

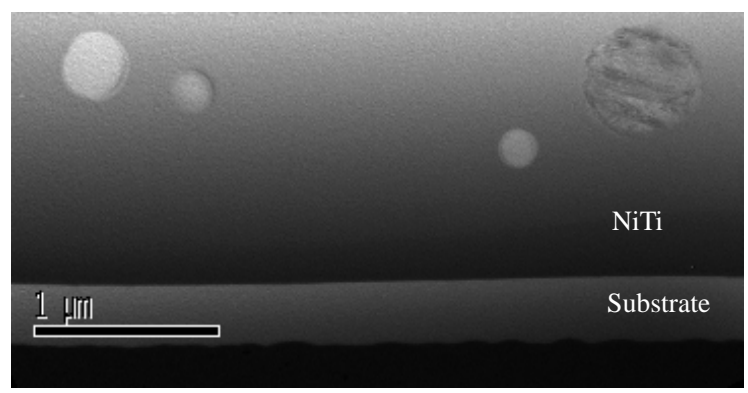

Fig. 5. Cross-section TEM of a partially crystallized NiTi film. 

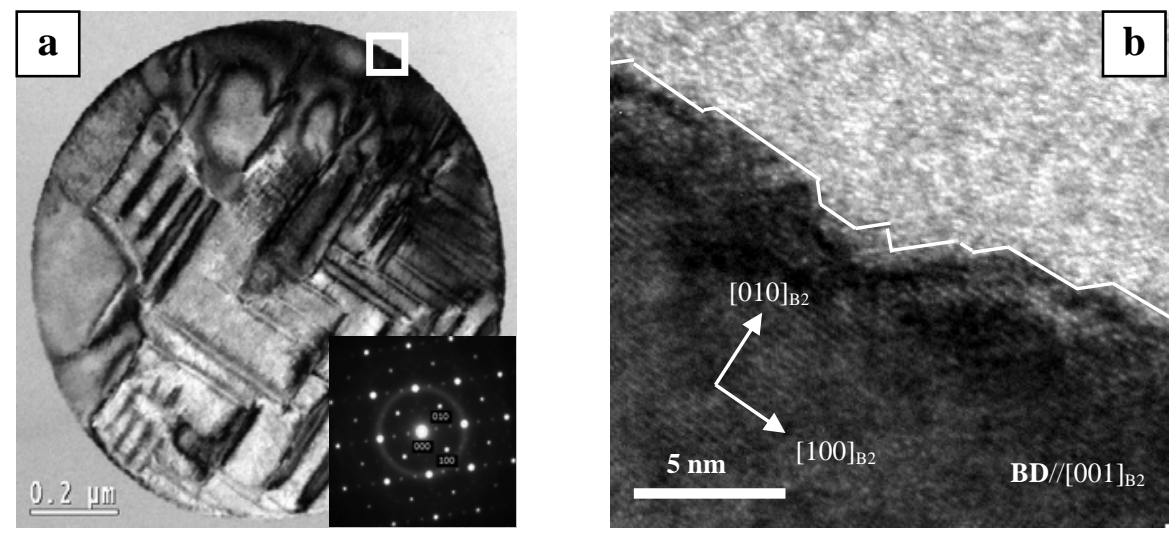

Fig. 6. (a) Low magnification electron micrograph showing two sets of mutually perpendicular needle domains in a grain. The inset electron diffraction pattern taken from both the matrix and needle domains shows a [001 $]_{\mathrm{B} 2}$-type zone with two sets of 1/3(110) superlattice reflections. (b) HRTEM image taken from the area indicated in (a), the trace of the interface marked by solid lines reveals a stepped growth interface along $\{100\}_{\mathrm{B} 2}$ and $\{110\}_{\text {B2 }}$ planes.

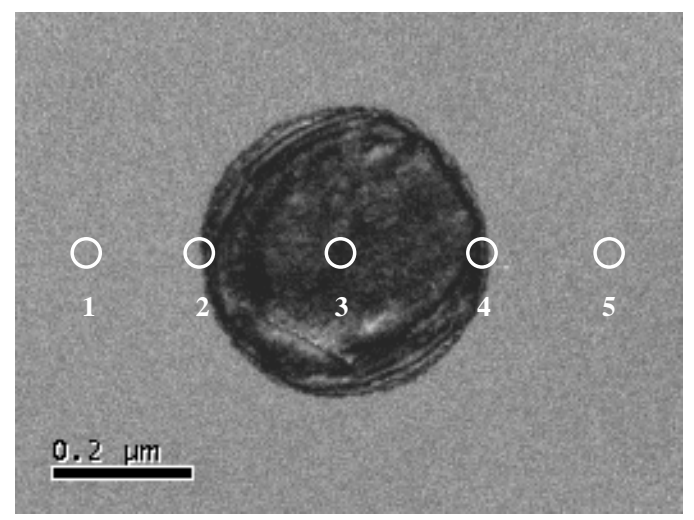

Fig. 7. Quantitative EDX analysis reveals no composition change upon crystallization (see Table 2). 


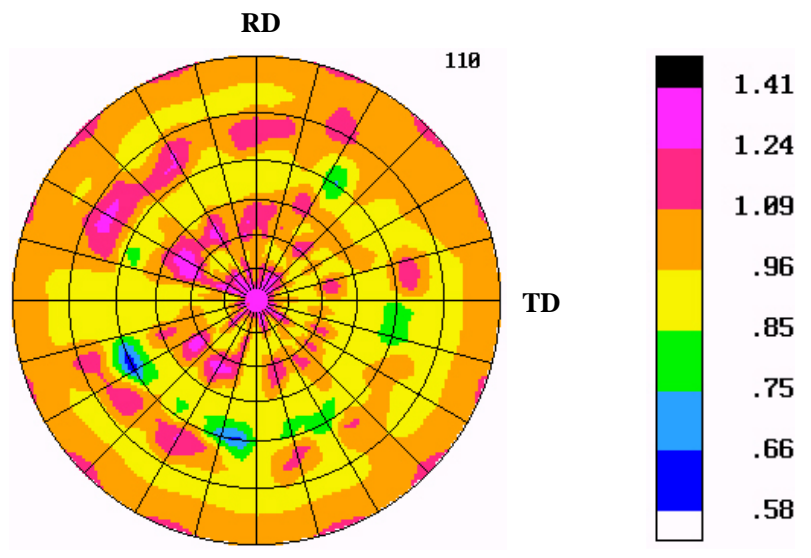

Fig. 8. $\{110\}$ pole figure for the laser annealed film. 

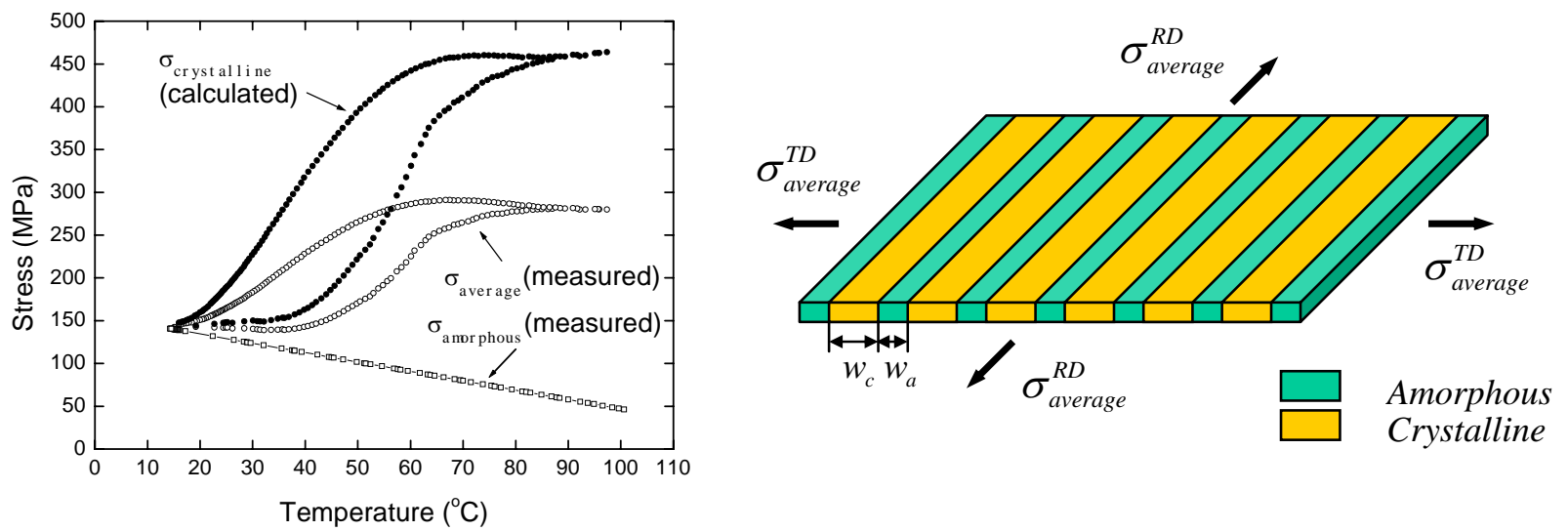

Fig. 9. Experimental stress-temperature curves for an amorphous specimen and a multiple-line specimen. The stress-temperature curve for the crystalline region was calculated from the experimental data using equation (1). 

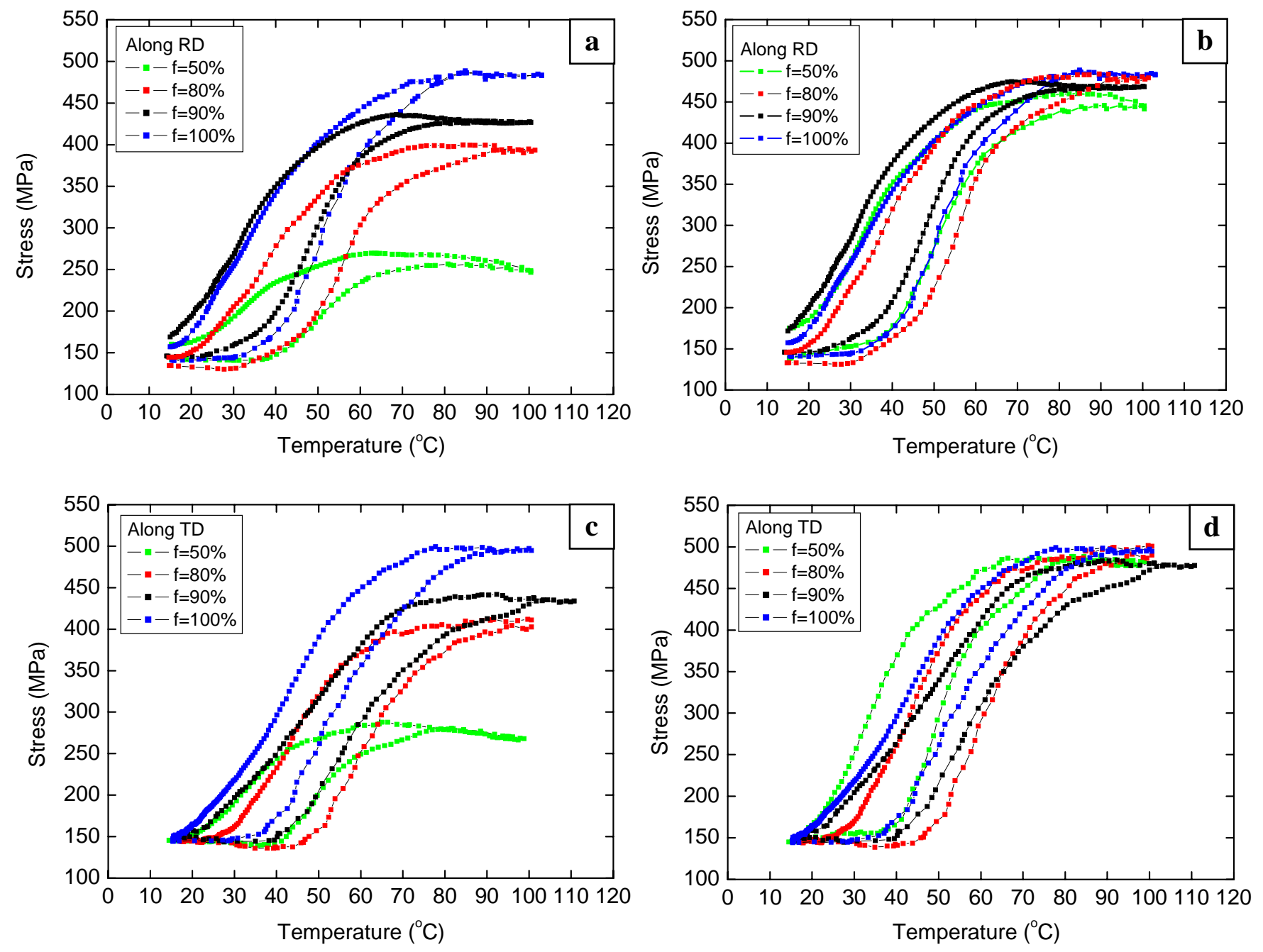

Fig. 10. The stress-temperature curves along the RD ((a) and (b)) and TD ((c) and (d)) directions for different laser annealing parameters. Figures (a) and (c) show the average stress in the specimens; figures (b) and (d) show the stress in the crystalline regions. 